Article

\title{
Enhancing Spatio-Temporal Identity: States of Existence and Presence
}

\author{
Pierre Hallot* and Roland Billen \\ Geomatics Unit, University of Liège, 4000 Liège, Belgium; rbillen@ulg.ac.be \\ * Correspondence: p.hallot@ulg.ac.be; Tel.: +32-4-366-5633 \\ Academic Editors: François Anton and Wolfgang Kainz \\ Received: 6 February 2016; Accepted: 2 May 2016; Published: 12 May 2016
}

\begin{abstract}
This work presents a new approach that aims to characterize the spatio-temporal relationships that exist between geographical objects that are absent or non-existent at the moment of analysis. First, we would like to propose a formal analysis of the spatio-temporal states of presence and existence of a geographical object. We will then use a combination of these states in order to define a set of life and motion configurations. The model developed then serves as a formal basis for the realization of a series of spatio-temporal queries based on an analysis of patterns in the succession of spatio-temporal states. The entire approach is then demonstrated by using the example of the organization of a scientific conference by defining the spatio-temporal relationships between the conference participants. The research methodology is finally compared with a real dataset taken from a geolocalized social network to show the efficiency of this type of management.
\end{abstract}

Keywords: spatio-temporal relationship; existence; presence; spatio-temporal request; time geography

\section{Introduction}

The search for and development of solutions using spatio-temporal data have greatly progressed over the last ten years following consistent improvements to data acquisition techniques and the evolution of the expectations and behavior of users. For example, whether this involves the use of active networks such as $\mathrm{Wi}-\mathrm{Fi}$, mobile phone network or satellite positioning systems, finding one's location is becoming easier and easier for anyone owning the right equipment. At the same time, the emergence of social networks has led users to share their position almost in real time. The emergence of these network location services has resulted in the availability of a large quantity of data. Despite these significant changes, we are still far from achieving an optimal use of this data located in space and time.

First of all, access to complete spatio-temporal information remains limited by constraints of quantity or availability of data, copyright policies or restrictions related to privacy laws. Analysis of spatio-temporal relationships is therefore often limited either to a given acquisition system or voluntarily limited to the scope of an event. Secondly, complex spatio-temporal reasoning often involves the implementation of past or future objects or events. Whether it involves a visit to the ruins of an old castle, a battlefield or, more simply, the planning of a future habitation, these objects or events are used as location markers or as bases for reasoning by defining spatio-temporal relations between one or several objects. In the same vein, it is not always possible to associate geometry or a spatial location at all moments of the life of an object. The object may not have or no longer have a geometry by nature as in the case of a proposed new or demolished building or due to a lack of data as in the case of a loss of a navigational signal for a car. Finally, the use of spatio-temporal data for the most part involves the use of semantics that are closely related to natural language. The queries performed on a spatio-temporal dataset are often seen to be complicated, stemming from the combination of a spatial and temporal line of reasoning. The perception of space-time requires a cognitive analysis 
of the perception of the final user in order to allow him to make requests that are close to his own language while enabling him to easily extract a wealth of information from a dataset.

In this article, we propose solutions for analysis that require continuous object's visibility or presence. Our objective is to make possible the management of spatio-temporal relationships between objects that may not yet have or no longer have a spatial extension and between objects that may not be present for a period of time while continuing to exist with regard to the system.

The article is structured in the following way. In Section 2, we draw up a state-of-the-art for spatio-temporal models dealing with the notion of identity of geographical objects. Then, in Section 3 , we define the concept of the spatio-temporal state as well as an ontological vision associated with it. Section 4 is devoted to the representation of life and motion configurations and their use in the formalization of spatio-temporal stories of geographical objects evolving in a space that does not offer the possibility of perfect localization. In Section 5, we propose to exemplify the model via analysis of the organization of a scientific conference. In this example we show the way to carry out spatio-temporal requests based on the search for patterns of successions of spatio-temporal states in life and motion configurations. The conference organization serves as an exemplar for model construction and for to build the model and to express all the possible diversities of performed queries. To remain close from a real dataset analysis problematic, we applied in Section 6 the research principles to a dataset taken from a geolocalized social network. Finally, we draw our conclusions and suggest possible future avenues of search.

\section{State-of-the-Art}

The identity of an object, which must not be confused with the "identifier key" concept [1], is the property intrinsic to each object which allows it to be differentiated from all others. In philosophy, this concept is known as the "identity of indiscernibles" [2]. The complete definition of a geographical object involves the definition of its identity and spatiality, which can vary over time. The identity of an object is defined as the unique characteristic which distinguishes it from all other objects [1].

Most of the work relative to modeling the identity of geographical objects is based on the modeling principle of unique object identity. This search, which includes that of [3-5] focuses on the concept of a geographical object and its modeling as a unique object. This work proposes a model dealing with possible transitions between geographical objects considering their identity and a formal modeling of the transformations applied to geographical objects taking account of their respective identities. Other models propose an analysis of the study of the evolution of geographical objects while considering their possibility to no longer exist. Campos [6] proposes a set of relationships and their associated semantics describing the representation of geographical objects in a virtual environment. Although they are semantically complete, these models do not take into account the possibility of studying a relationship between an object present at the moment of the analysis as against an object that existed in the past or an event existing in the future. For example, it is not possible for these models to search all the people visiting a future habitation site.

The study of relations between regions evolving in space such as that proposed by Claramunt [7-9] makes it possible to define a set of spatio-temporal relationships by integrating spatial topological reasoning $[10,11]$ with the temporal logic proposed by Allen $[12,13]$. These works propose studying the sequences of change during the evolution of cadastral parcels [14]. More recently, other models of spatio-temporal reasoning have sought to describe the trajectories of geographical objects in an allocentric [15-17] or egocentric vision [18,19]. These models, based on a spatio-temporal mereology, describe, by means of qualitative operators, the relationships that exist between moving objects. This paper focuses on an approach where all the objects exist at every moment. Modeling of spatio-temporal information is a very active field of research; a comprehensive state-of-the-art is out of the scope of this paper. Interested reader can refer to [20-24] for a survey of existing works.

One of the objectives of a spatial or spatio-temporal reasoning model is to formalize the semantic links which bind objects or events in space and time [25-27]. The study of the semantics of spatio-temporal relationships makes it possible to obtain queries that are closer to the perception 
of final users, to construct models for analyzing natural language, and to link spatial and temporal concepts of language with a formalism that allows analysis [28,29].

The notion of geographical context [30], which represents the environment perceptible to one or several users maintains strong links with the proposed research. The perception of the presence or absence of a geographical object calls into question the context in which the object is observed. An omniscient user sees all the information relative to a given geographical location. Any other user will only see part of this reality, restricted by the context in which he observes it.

Currently, analysis of the temporal and spatial evolution of one or several geographical objects focuses either on the description of its trajectory or the relationships that several objects have during their displacement, or on the appearance or disappearance of the objects in time based on their identity. The description of the movements of a mobile phone based on qualitative operators is limited to periods of time during which an object can be observed. The proposition of a model combining the possibility of analysis of spatio-temporal connections between objects including the possibility of their non-existence or non-presence during a period of time makes it possible to bring these two approaches together under the guise of a more general unique model [31]. The model proposed thereafter, responds to this assessment and makes possible a more general analysis of all the situations concerning the evolution of geographical objects in time and space.

\section{Spatio-Temporal States}

Let us postulate that a geographical object cannot exist for an infinite amount of time. In other words, there will always be a period of time during which the object does not yet exist and a period during which the object will no longer exist. Moreover, we also postulate that an object that has been destroyed and whose identity has also been destroyed cannot appear again in the same form, i.e., we do not accept the resurrection of the geographical object. The stage that marks the creation of an object is defined by the thought experiment that aims to conceptualize it. However, it would not be foreseeable to conceive of a system that makes it possible to materialize every geographical object created during a thought experiment. This is why it is preferable to restrict this definition by postulating that the existence of an object begins as soon as a semantic or spatial relationship is established for this object and that it ceases to exist when there are no more relationships with it. In order to make our position clearer, we begin with the following examples. A dwelling has an identity as soon as it has been subjected to a thought experiment (the creator of the work envisions the creation of a dwelling) and that the relations are established with the object and other existing objects (a building plan begins to be drawn up, a request for planning permission is submitted for the future construction). At this moment in time, the physical construction of the dwelling does not yet exist but its identity is well and truly real in the sense that it is used for communication purposes or as an object in a legal procedure. Furthermore, it may happen that an object is created by the definition of its identity and links to this object but that no physical construction occurs in time. We think that, in this case, it is always useful to speak about a geographical object because although it only has one virtual extension into the physical world, this can be used as a reference point or serve as a basis for spatial reasoning. An example of this scenario is the case of a group of individuals manifesting their opposition to a construction project that will later be abandoned. A possible request would be to search for all the people present on the construction site in order to identify the protesters. Here, we are in the presence of a relationship between existing objects (the protestors) and a reference object that does not have a physical realization (the construction project).

When an object exists, it is not necessarily always recognizable during its entire existence. Whatever the type of monitoring of geographical objects, there are two periods of time during which the object is spatially outside the analysis zone (for example, when an individual is outside the reach of a network of active sensors), or periods during which the acquisition system does not make it possible to obtain information with regard to the spatiality of the object (for example, when a vehicle followed by a satellite positioning system is in a tunnel). These different cases do not entirely correspond to a lack of information or vague information because the identity of the object very often remains known. Loss of information about the temporary spatial extension does not destroy the existence of the geographical object. 
In order to take account of these various possible states of a geographical object, we propose to formally define the different possible states with regard to a line of spatial, temporal and object reasoning.

\subsection{Temporal Line of Reasoning}

The temporal line of reasoning used refers to Galton's proposition [32]. This reasoning makes it possible to effect the connection between the two paradigms of temporal representation that are instants and time intervals.

Let us postulate that $\mathcal{T}$ is a set of instants. We therefore consider the following temporal logic $\mathcal{T}=(T,<)$ possessing the characteristics of irreflexivity (Equation (1)); transivity (Equation (2)); linearity (Equation (3)); no end (Equation (4)); and density (Equation (5)).

$$
\begin{gathered}
\forall t \neg(t<t) \\
\forall t, u, v(t<u<v \rightarrow t<v) \\
\forall t, u(t \neq \mathrm{u} \rightarrow t\langle u \vee t\rangle u) \\
\forall t \exists u, v(u<t<v) \\
\forall t, u(t<u \rightarrow \exists v(t<v<u))
\end{gathered}
$$

The temporal interval is defined based on instants. Let us consider $\mathscr{J}$ as a set of temporal intervals. For each pair of distinct instants $t$ and $u$, there exists an interval I composed of all the instants from $t$ to $u$ inclusive. In addition, $t$ and $u$ are called the beginning and the end of the time interval respectively (Equation (6)). Furthermore, Galton also proposes the possibility of returning to Allen's temporal relationships [13] based on this set of axioms [33].

$$
\forall t, u(t<u \rightarrow \exists I \wedge \forall i((t \leqslant i \leqslant u) \rightarrow i \in I))
$$

A theory of states can be superimposed on the temporal logic. This serves to link the temporal primitives with the objects. We use the predicate Hold-at Equation (7) to link the states to instants and the predicate Holds Equation (8) to define the state of a valid object during a time interval. With $S$, a state, $i$, a time interval and $t$ an instant, we obtain:

$$
\begin{aligned}
& \text { Holds }-a t(S, t) \equiv \text { state } S \text { holds at the instant } t \\
& \text { Holds }(S, i) \equiv \forall t(\operatorname{Div}(t, i) \rightarrow \text { Holds }-a t(S, t)
\end{aligned}
$$

with $\operatorname{Div}(t, i)$, which signifies that the instant $t$ is strictly included in the interval $i$.

\subsection{Workspace}

The originality of the approach consists of defining the concept of workspace. A workspace represents that portion of space in which a phenomenon is studied. This space is defined with the help of presence functions. Note that the concepts defined here are not limited to geographical spaces but that they can certainly be transposed to analyses of virtual spaces such as social networks, legal domains, mathematics, etc. The workspace can represent either the space for analysis of a geographical object or a composition of geographical objects such as the spatiality of an event.

$$
W_{t}=\{x \in \text { space }: P(x, t)
$$

In the definition Equation (9), space represents the infinite set of all the possible positions for an object and $P(x, t)$ the set of functions of presences defining whether an object is situated in the workspace. The presence functions are also dependent on time. In fact, a workspace can change its spatiality over the course of time. 


\subsection{Spatio-Temporal State}

The spatio-temporal state of a geographical object is conceptually defined as the combination of the existence and presence of a geographical object. The concept of state refers to Galton [34] and is defined as the set of all object's properties and components that can remain stable (hold) at an instant or during an interval of time. In order to take account of the different spatio-temporal states of an object, we propose a conceptual representation of the different concepts that come into play during their definition (Figure 1). The first state of an object or an event is that of non-existence. While this state cannot be modeled in a computer system, it represents the first stage of the conceptualization of the entity A; it concerns the thought experiment of object A. The definition of object A comes into play when a relation is established between this object and another object or event which is itself already defined. An identity is then attributed to the object or event A, it enters a spatio-temporal state of existence. Two possibilities are then possible. In both cases, a portion of space is defined as corresponding to the location of object A. If the object is materialized in this portion of space, it is described as present. It will be said to be absent if it is realized in space; that is to say, that the space is defined but is not physically present there, the stage allowing for the transition of these two states is described as spatialization.

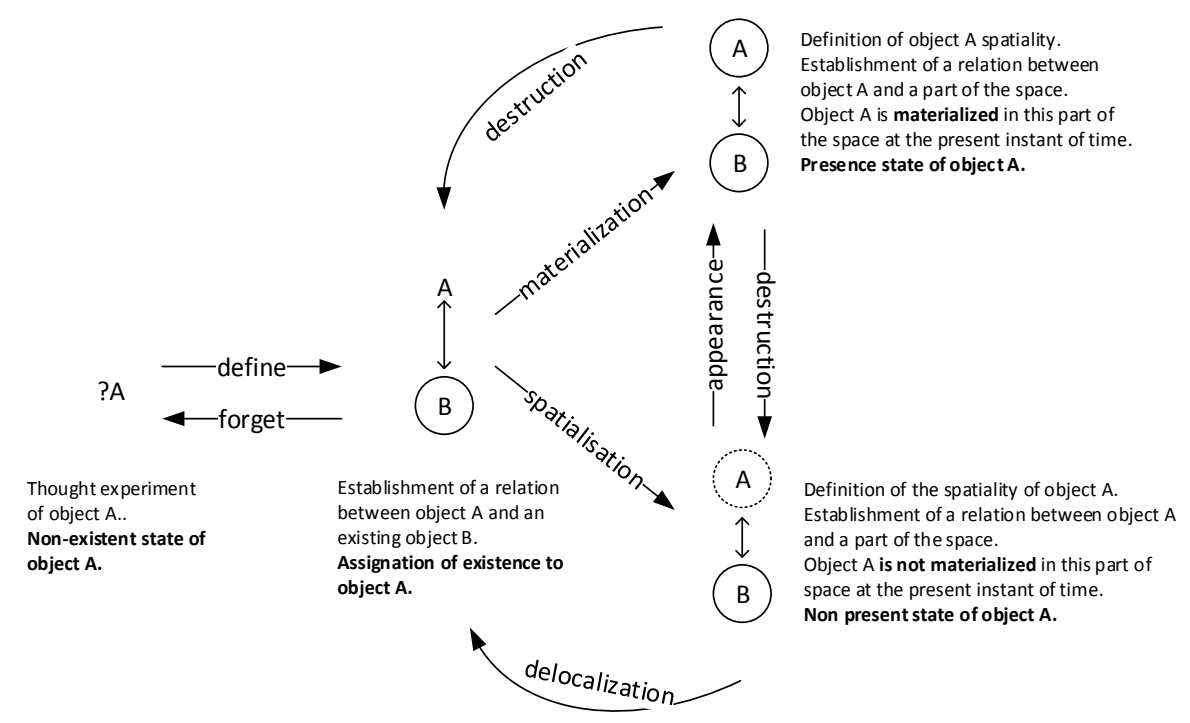

Figure 1. Conceptual schematization of the different spatio-temporal states of an object or event A. The arrows represent the transition events between these different states. The destruction corresponds to an omission of the location of the object, the delocalization marks the loss of the realization in the physical space without omission of its position.

The spatio-temporal state of existence of an object or event $\mathrm{A}$ is defined as:

$$
\forall j \in I, \exists i_{B} \in \mathscr{J}, \exists R_{B}-A_{A} \text { Aspace } \rightarrow \exists i_{A} \in \mathscr{J} \wedge \text { Hold }-a t(\exists A, j)
$$

With $\mathrm{j}$ a temporal instant, $i_{B}$ the identity of an object $\mathrm{B}$ and $R_{B}$ - the relation that links object $\mathrm{B}$ and object $\mathrm{A}$. We will use the notation $\exists A$ in order to describe the spatio-temporal state of existence.

The spatio-temporal state of presence, denoted as $p A$ is defined as:

$$
\forall i_{A} \in \mathscr{J}, \forall j \in I: A \subset_{j} S \rightarrow \operatorname{Hold}-a t(p A, j)
$$

with $i_{A}$ the identity of object $\mathrm{A}, \mathrm{j}$ a temporal instant, and $S$ the space. We will use the notation $p A$ in order to designate the state of a geographical object that is present; that is to say, whose spatiality is accessible at the instant of analysis. 
The spatio-temporal state of non-presence or absence is defined as:

$$
\forall i_{A} \in \mathscr{J}, \forall(j, k) \in I: \mathrm{j} \neq \mathrm{k}, A_{j} S \wedge A \subset_{k} S \rightarrow \operatorname{Hold}-a t(n p A, j)
$$

with $i_{A}$ the identity of object $\mathrm{A}, \mathrm{j}$ and $\mathrm{k}$ two instants and $S$ the space. The state of absence is therefore defined as the state of an object possessing an identity but not included in the space at the present instant. We add the constraint that the object is part of the space at a future or past instant $k$.

\subsection{The Spatio-Temporal State and the Workspace}

The workspace in which a geographical object evolves can be compared to the notion of geographical context [30]. If we consider an allocentric view, it indicates the visibility part of the analysis, i.e., it responds to the question: "what portion of space is at the disposal of my analysis"? An egocentric vision in relation to a geographical object answers the question: "what portion of the space is within my possibility for actions". This second vision of things brings the notion of workspace closer to the study of spatio-temporal prisms and many works based on Hägerstand [35].

Following this work, we will concentrate on analysis of the geographical state of an object with regard to whether or not it belongs to a workspace. We therefore postulate that the definitions Equations (11) and (12) can be restricted by considering that they belong to the workspace $W$ and no longer to the total space $S$. This leads us to define two supplementary states depending on the activity of the workspace. In the case of an active workspace, we obtain:

$$
\begin{array}{r}
\forall j \in I, \exists i_{B} \in \mathscr{J}, \exists R_{B}-A, A W \wedge W \subset_{j} S \rightarrow \exists i_{A} \in \mathscr{J} \wedge \text { Hold }-a t(\underline{\exists A}, j) \\
\forall i_{A} \in \mathscr{J}, \forall j \in I: A \subset_{j} W \wedge W \subset_{j} S \rightarrow \text { Hold }-a t(\underline{p A}, j) \\
\forall i_{A} \in \mathscr{J}, \forall(j, k) \in I: \mathrm{j} \neq \mathrm{k}, A_{j} W \wedge A \subset_{k} W \wedge W \subset_{j} S \rightarrow \text { Hold }- \text { at }(\underline{n p A}, j)
\end{array}
$$

In the case of an inactive workspace, we obtain:

$$
\begin{gathered}
\forall j \in I, \exists i_{B} \in \mathscr{J}, \exists R_{B-A}, A W \wedge W_{j} S \rightarrow \exists i_{A} \in \mathscr{J} \wedge \operatorname{Hold}-a t(\exists A, j) \\
\forall i_{A} \in \mathscr{J}, \forall j \in I: A \subset_{j} W \wedge W_{j} S \rightarrow \operatorname{Hold}-a t(p A, j) \\
\forall i_{A} \in \mathscr{J}, \forall(j, k) \in I: \mathrm{j} \neq \mathrm{k}, A{ }_{j} W \wedge A \subset_{k} W \wedge W_{j} S \rightarrow \operatorname{Hold}-a t(n p A, j)
\end{gathered}
$$

Visually, the relations Equations (13)-(18) can be represented according to Figure 2. We will use a representation of temporal space to visualize a workspace $W$ as well as the four corresponding spatio-temporal states.

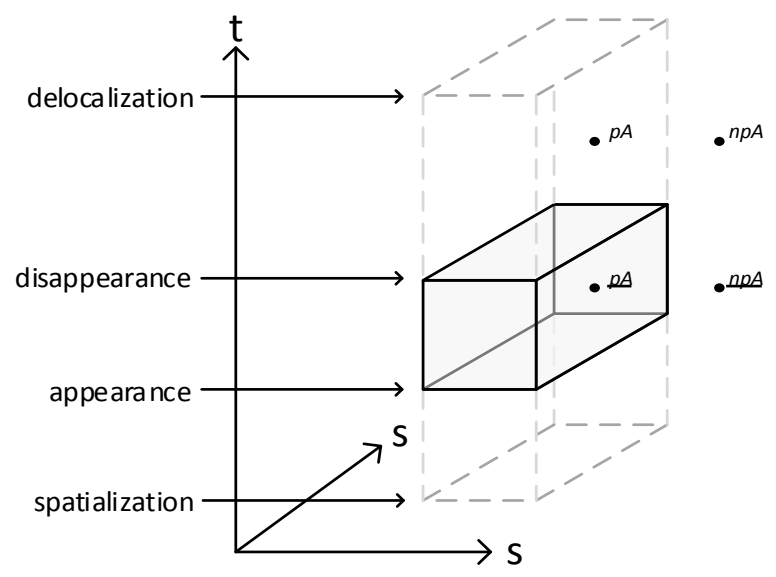

Figure 2. Representation in a temporal space of 4 spatio-temporal states applied to an active workspace (unbroken region) and inactive (dotted region). The transitions between states of activity in the workspace are described by the transition events. 
A complete study of possible transitions between spatio-temporal states was conducted by Hallot et al. [31,36]. This resulted in the representation of a conceptual diagram of the neighborhood [37] justified by dominance theory [38]. Some applications where the user perspective is considered to be omniscient may require considering an unlimited workspace in space, time or both. For such cases, all the possible spatio-temporal states are not possible. An unlimited workspace in space will always imply a visible object. This degeneration of the proposed model can lead to classical view of information (without considering the possibility of not being present or not existing during a period of time).

\section{Life and Motion Configuration}

Follow-up analysis of spatio-temporal relations between geographical objects in the context of a workspace is carried out by means of life and motion configurations. Life and motion configurations are the formal expression of spatio-temporal stories with regard to the application of the theory of states defined previously. The construction of all the life and motion configurations involves determining the spatio-temporal relations between two objects. In order to do this, we propose to combine the spatio-temporal states of two objects with regard to a workspace. We will then study the possible outcomes for these different states in order to search for all the possible outcomes between two objects.

\subsection{Relations between Spatio-Temporal States}

The relations between spatio-temporal states are defined as the product of the two sets of spatio-temporal states for an object. There are 36 relations between spatio-temporal states. For each of these, semantics can be attributed. Here we will describe in detail some significant examples. We note that the spatial relations between two objects can only be studied when both of them possess a spatiality within a workspace. The relations between spatio-temporal states can therefore be seen as the doorway to a reality of geographical objects that have not yet been modeled.

The spatio-temporal states relationships are obtained from the cross product of the individual spatio-temporal states defined in a same workspace (Equation (19)).

$$
\operatorname{STS}_{W}(\mathrm{~A}-\mathrm{B}) \triangleq \operatorname{STS}_{W}(\mathrm{~A}) \otimes S T S_{W}(\mathrm{~B})
$$

The set of the 36 relations between spatio-temporal states can be ordered in a matrix $(6 \times 6)$. The identical spatio-temporal states are located on the matrix diagonal. Equation (20) shows a dense representation of possible spatio-temporal states relationships.

$$
\operatorname{STS}_{W}(\mathrm{~A}-\mathrm{B}) \triangleq\left[\begin{array}{ccc}
\exists \mathrm{A} \exists \mathrm{B} & \cdots & \exists \mathrm{A} n p \mathrm{~B} \\
\vdots & \ddots & \vdots \\
\underline{n A} \exists \mathrm{B} & \cdots & \underline{n p A n p B}
\end{array}\right]
$$

\subsection{Analysis of Relations between Spatio-Temporal Stories}

The model of life and motion configurations can also be extended to take account of spatial relations, such as the topological relations between regions [10] or projective relations [39]. Following our analysis, we will only take account of a relation of inequality between two geographical objects. The relation between two spatio-temporal stories is described as a succession of relations between spatio-temporal states. The different outcomes possible were studied by Hallot [40] in order to ensure that all the relations between spatio-temporal objects are described in the life and motion configurations. Figure 3 describes the spatio-temporal relations between two objects moving inside and outside a workspace. As a reminder, their presence and absence are defined by the fact of being part of the workspace and the activity of the workspace is described by whether or not the relation is underlined. Figure 3 shows three examples of the evolution of two objects assimilated to points in a temporal space. Each relation between spatio-temporal states is described in the life and motion configuration, noted as 
LMC, associated with each example. Subsequently, analysis of the spatio-temporal phenomena will be carried out. This analysis will be based directly on the study of the life and motion configuration instead of analyzing the form of the spatio-temporal story. Writing the relation between spatio-temporal stories in the form of life and motion configurations can be seen as a generalization of these stories by focusing on the relations of identity and presence of the objects analyzed.

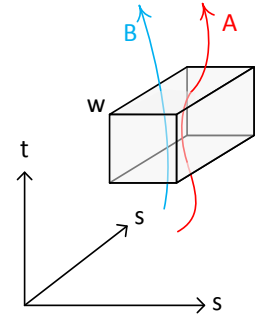

$\operatorname{LMC}_{W}(A-B)=$

$\{p A \exists B$,

$\mathrm{pApBdis,}$

$\mathrm{pApBdis}$

$\mathrm{pApBdis} \mathrm{\}}$

(a)

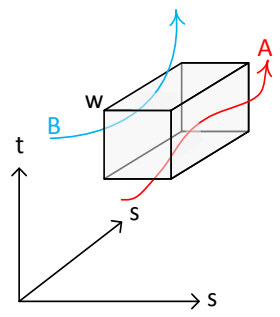

$\operatorname{LMC}_{W}(\mathrm{~A}-\mathrm{B})=$ $\{p A \exists B, p A \exists B$, pAnpB, npAnpB, npApB, npApB, $\exists \mathrm{ApB}\}$

(b)

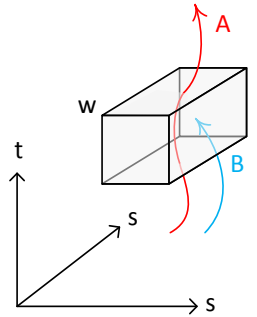

$\mathrm{LMC}_{\mathrm{W}}(\mathrm{A}-\mathrm{B})=$

$\{p A n p B, p A p B$,

$\mathrm{pA} \exists \mathrm{B}, \mathrm{pA} \exists \mathrm{B}\}$

(c)

Figure 3. Three examples of the description of relations between spatio-temporal stories by means of life and motion configurations: (a) two objects located in the workspace before, during and after its activity; (b) object A leaving the workspace during its period of activity while B arrives later at the beginning of the workspace activity; and (c) an object B whose existence ceases during the activity of the workspace.

The examples proposed up to the present relate to only one workspace, however, the combination of several workspaces is conceivable. When the analysis carried out is based on several methods of acquisition providing a heterogeneity in the acquired spatiality and temporality of objects, for example. It is possible to imagine the combination of a spatial acquisition by means of a network of active sensors by satellite positioning. Another option for a combination of workspaces is to work on the granularity of objects and spatio-temporal relations. Indeed, if the workspace corresponds to an office environment, it is possible either to analyze the presence of objects only with regard to the position within the building, or to detail the analysis in relation to the offices themselves. In this case, spatial and temporal relations that will exist between workspaces could provide information about a possible deduction about the spatio-temporal state of the objects present. For example, if an individual is present in his or her work office, he or she is considered to be present in the building where the office is situated. On the other hand, if an individual is absent from any office but present in the building, we can conclude that the individual in question is present in a common area of the building. This property also makes it possible to predict what the future spatio-temporal states are or what the relations between spatio-temporal states are for one or two objects.

\subsection{Interpretation of Life and Motion Configurations}

Modeling the spatio-temporal states of an object and its relations make it possible to mainly carry out requests in the form of the search for motifs in life and motion configurations. We think that this method of analysis makes it possible to easily find a set of objects answering a particular search criterion. This method of working also makes it possible to associate predefined outcomes for relations between spatio-temporal states with concepts of natural language. In this way, we think we can supply a work context that makes it possible to develop an understanding of requests formulated in natural language. Semantic search is currently the object of very particular analyses [41] in particular, in search related to the semantic web and inferences based on ontologies [42]. In order to fully understand the queries based on life and motion configuration patterns recognition, the defined predicates will be applied to an example of a conference organization in the next chapter. 
We have selected a set of predicates that are easily transferable in the context of an analysis of life and motion configurations. Note that we do not intend to present an exhaustive list of all the semantic relations it is possible to get based on analysis of motifs in life and motion configurations. Rather, we propose a work context in which a user can easily find the relation he is searching for. It is also obvious that all the semantic relations cannot be explained by means of the proposed model. However, as this is extensible by means of a spatial relation and the fact that it takes account of more generic cases than the models cited in the state-of-the-art, we think it can be used for several domains and analyses. The concepts we propose to analyze are the following: meeting, crossing, generation, disappearance, ignorance and precedence. For each term, we give our expression of the semantics within it as well as the life and motion configuration that corresponds to it.

\subsubsection{Meeting}

Meeting is seen as the fact that two objects meet during a time interval at the same spatio-temporal point. It is to be differentiated from crossing for which temporality is linked with an instant.

$$
\forall L M C_{W}(A-B): \exists\{\ldots, \text { Holds }(\underline{p A p B e}, i), \ldots\} \rightarrow \text { A meets } B
$$

where $i$ is a time interval.

Equation (21) expresses the fact that during the life and motion configurations, we should find the spatio-temporal relation mentioned. The notion of meeting can be broken down into four states if the acquisition of the spatiality of objects is not dependent on the workspace. In this case, we could detail the semantics of the meeting in a more precise way. We would get, for example:

$$
\forall L M C_{W}(A-B): \exists\{\ldots, \text { Holds }(p A p B e, i), \ldots\}
$$

$A$ meets $B$ in the future or previous workspace.

$$
\forall L M C_{W}(A-B): \exists\{\ldots, \text { Holds }(\mathrm{n} p A n p B e, i), \ldots\}
$$

$A$ meets $B$ outside the future or previous workspace.

$$
\forall L M C_{W}(A-B): \exists\{\ldots, \text { Holds }(\underline{p A p B e}, i), \ldots\}
$$

$A$ and Bmeet in and during the workspace activity.

$$
\forall L M C_{W}(A-B): \exists\{\ldots, \text { Holds }(\underline{n p A n p B e}, i), \ldots\}
$$

$A$ and $B$ meet outside the workspace during the activity of the workspace.

The refinement proposed by the detail of the location cases or the lack thereof in the activity of the workspace depends on the level of granularity of the analysis carried out. Following this, we will nonetheless consider that the acquisition of the geometry of the object depends on its participation in the workspace.

\subsubsection{Crossing}

Crossing is seen as the spatio-temporal meeting of two objects that do not have a time duration; that is to say, occurring during a moment of time. Formalization is very close to the Equation (20) even if only because the predicate used to represent the relation between spatio-temporal states is not Holds but is, in fact, Holds-at, expressing a state that is valuable for an instant. 


$$
\forall L M C_{W}(A-B): \exists\left\{\begin{array}{c}
\text { Holds }(\underline{p A p \mathrm{Bd}, t}), \\
\text { Holds }-a t(\underline{p A p \mathrm{Be}}, u) \\
\text { Holds }(\underline{p A \mathrm{pBd}}, v), \\
\ldots
\end{array}\right\} \rightarrow A \text { crosses } B
$$

where $t$ and $v$ are two time intervals, and $u$ is an instant. The theory of dominance indicates to us that in this case, only the state $p A p$ Be can be instantaneous.

The same refinement as mentioned previously can be applied in order to specify whether the crossing took place or not in the activity of the workspace.

\subsubsection{Generation/Creation}

Generation consists in seeing the appearance of an object during the period of presence of a second object. The first position of the object that appears must be spatio-temporally identical to the object that generates it. The motif corresponding to this concept is:

$$
\forall L M C_{W}(A-B): \exists\left\{\begin{array}{l}
\ldots, \text { Holds }-a t(\underline{p A \exists \mathrm{B}}, t), \\
\text { Holds }-a t(\underline{p A p \mathrm{Be}}, u) \ldots
\end{array}\right\} \rightarrow \text { A generates } B
$$

\subsubsection{Capture}

Capture is seen as the inverse relation of generation. We interpret the concept as the fact that an object loses its spatiality just after a meeting or crossing with another object. The motif is expressed as follows:

$$
\forall L M C_{W}(A-B): \exists\left\{\begin{array}{c}
\ldots, \text { Holds }-a t(\underline{p A p \mathrm{Be}}, t) \vee \operatorname{Holds}(\underline{p A p \mathrm{Be}}, u), \\
\operatorname{Holds}(\underline{p A \exists \mathrm{B}}, v) \ldots
\end{array}\right\} \rightarrow \text { A captures } B
$$

where $t$ is an instant, and $u$ and $v$ are time intervals.

\section{Life and Motion Configurations Applied to the Organization of a Conference}

In the rest of this section, we propose to construct an example of the use of life and motion configurations in order to give an account of their efficacy in the context of analyzing a geographical phenomenon for which the environment is not constant over the course of time. This scenario is proposed to encompass the whole diversity of results obtained from the application of life and motion configuration.

The organization of a scientific conference can be defined in terms of a succession of several events linked to a principal event which is the conference. Most conferences are cyclic events, in the main, they take place annually or bi-annually. In the rest of our analysis, we will focus on the elements of connection between the participants and the edition of a conference. The geographical event, the conference, is composed of an identity, a spatiality and a temporality. We think that the first stage of the definition of a conference is the definition of its identity. As previously explained, the conference can only be explained by a system when a participant communicates something about its identity and creates relations between this and other participants. In particular, it is when a member of a group of scientists communicates information about the organization of the next conference in a given field. From the moment a communication relative to the event is established between several participants, the identity of the conference is defined. However, no information is as yet defined with regard to its location or exact temporality, i.e., the date and place of the conference are not yet known. Given that only the identity of the conference is known, its state is therefore $\exists \mathrm{W}$ for a conference $\mathrm{W}$. The evolution of the definition of the event then takes place by means of the definition of a future location and future 
temporality of the activity, i.e., the place and time of the conference are fixed. The representation of the spatio-temporal story of this event corresponds to the succession of spatio-temporal states associated with the event:

$$
\text { Conference } W=\{\exists W, n p W, p W, \underline{p W}, p W, n p W, \exists W\}
$$

In a general way, the complete life of every geographical object or every geographical event takes place by means of these stages of spatio-temporal states. The difference between the different situations resides in the fact that certain spatio-temporal states are instantaneous or valid during a time interval. The semantic description of the conference event described by the succession of spatio-temporal states Equation (29) can be expressed as follows:

- $\quad \exists W$ : The identity of the conference is defined and relations with other objects or events are established.

- $n p W$ : The spatiality of the conference is defined but not yet active or present. At this stage, only the location of the conference is defined, nothing materialises this particular event into the physical world.

- $\quad p W$ : The conference is present but not yet active. This stage corresponds to the preparation of the conference. The physical relation of the conference appears. The markings indicating the location are put in place, the layout of the rooms materializes the conference, and the edifice in which the conference will take place is constructed.

- $\quad p W$ : The conference has materialized and is active. This is the time interval during which the

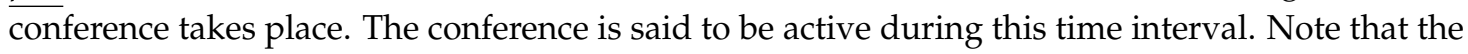
application of the theory of dominance demands that this state cannot be instantaneous. If the activity of an object or geographical event appears, this happens during a time interval. As stated previously, it is possible that this state never exists in the succession of spatio-temporal states of an object. This would be the case where the conference had been cancelled.

- $\quad p W$ : The conference is no longer active but is still present. This stage corresponds to the dismantling that is identical to the construction of the location of the conference. The difference between the setting up and dismantling will be done by means of analysis of the succession of spatio-temporal states of the conference, i.e., if the spatio-temporal state precedes or succeeds the state of presence and activity.

- $\quad n p W$ : This spatio-temporal state corresponds to the non-presence of the conference. Nothing else materializes the event in the physical world. However, the spatiality and identity of the conference (its identity) is still defined. However, the participants still refer to the conference (its identity) and to the location of this place (its spatiality).

- $\quad \exists W$ : At this stage, the spatiality of the conference is forgotten. There is no longer any relation existing between the conference and its spatiality. The only information still in memory (towards which relations exist) is that regarding its identity.

The workspace of a scientific conference also varies during time. Figure 4 expresses this spatial variability in the workspaces. The left side represents the situation where a conference finishes by a meeting that took place in a restricted space with regard to the entire area covered by the conference. For example, it is a workshop organized at the end of a conference but which only uses one part of the building. The right side represents the recurrence observed during conferences organized each year at the spatial location.

Based on this context, we define the spatio-temporal states of an individual in accordance with the workspace defined by the conference. The identity of an individual is studied with regard to his knowledge of the conference, i.e., the workspace. An individual who has registered for the conference has an identity with regard to it. Its spatiality does not yet exist at this stage. As soon as an object exists, it can then be located and a link to its spatiality is established. This spatiality is then considered with regard to the workspace defined by the conference. The different possible spatial states are represented in Figure 5. An individual is seen to be in relation to the workplace in four main ways with regard to the workspace defined by a conference. 
- $\quad p A$ : The individual $\mathrm{A}$ is spatially located in the space defined for the conference but during the inactivity of the latter. This signifies that the individual is present at the place where the conference takes place before it starts or after it finishes.

- $\quad n p A$ : The individual $\mathrm{A}$ is temporally existent during the activity of the conference but not spatially located in the space defined by the latter. It is a person who is known to the organization, whose identity exists, but not located in the space defined by the conference after or before its activity.

- $\quad p A$ : The individual $\mathrm{A}$ is temporally and spatially present during the conference. It is a Person who attends the conference.

- $\quad n p A$ : The individual $\mathrm{A}$ is not spatially located in the workspace of the conference but he is temporally present during its activity. This situation is the case of an individual who is going to join the conference after it starts.
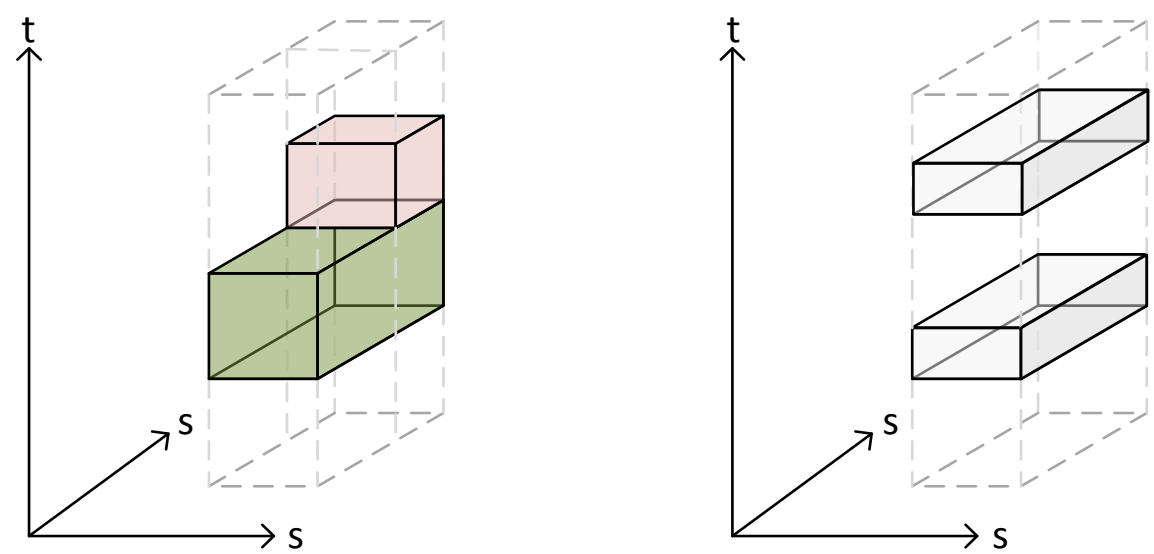

Figure 4. The temporal evolution of workspaces relative to the organization of a scientific conference. The left side represents a reduction of the space in which the conference is organized, e.g., a workshop directly following the conference but which only occupies one part of the building. The right side represents the temporal succession of a conference located in the same space.

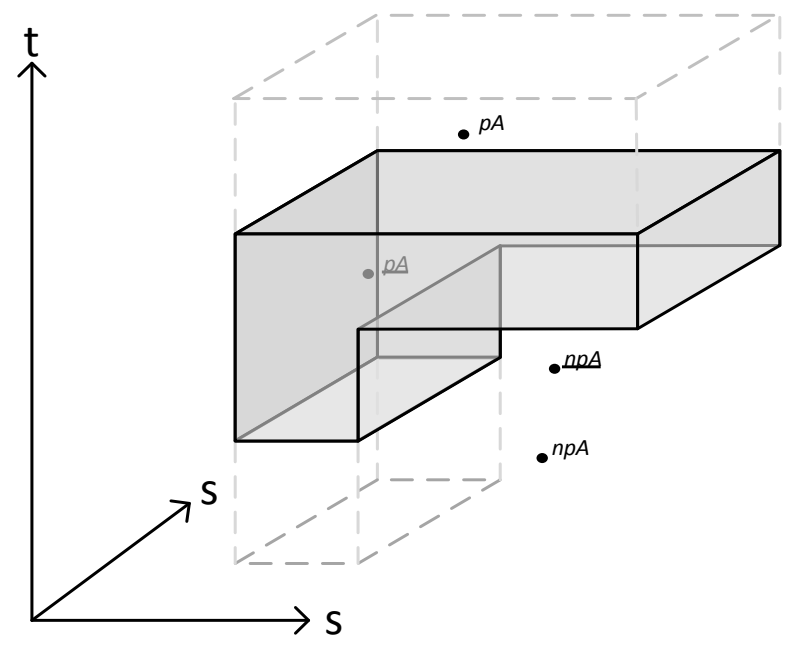

Figure 5. Symbolization of the four possible spatio-temporal states of an individual with regard to the context of the organization of a conference, considered as a workspace. An individual can be present during the activity of the conference $(p a)$, present during the period of activity of the conference $(p a)$, non-present during the occurrence of the conference ( $\underline{n p a)}$, and finally, non-present during the inactivity of the conference (npa). 


\subsection{Requests Relative to an Individual with Regard to a Workspace}

Given that the workspace of the conference and the semantic link between the different spatio-temporal states of an individual participating in the conference have been defined, it is now possible to study the evolution of an individual with regard to the conference in terms of successions of possible spatio-temporal states and to define search motifs corresponding to a request for the interaction between an individual and the conference. Considering a perfect location for each individual in relation to the conference, the visualization of each spatio-temporal story can only inform a small part of a particular spatio-temporal succession of each individual. It would be difficult to visually search for all the individuals who are present in the place the conference is taking place before it begins or individuals who leave the conference while it is in progress, for example. By studying a chosen succession of spatio-temporal states, we can easily identify each individual corresponding to particular semantics of movement. We will then describe the different possible movements of an individual and we will give the succession of spatio-temporal states corresponding to them.

- Which individuals arrive in place before the start of the conference? The search for people arriving in place before the beginning of the conference can provide information about their quality as organizers or participants. In fact, we can consider that the organizers will be spatially present during the beginning of the activation of the conference workspace. In order to search all the individuals corresponding to this category, it is necessary to search for all the individuals who possess an identity with regard to the conference, who are located in the location before the conference starts and, finally, who remain in place until the beginning of the conference. In terms of successions of spatio-temporal states, this brings us back to searching for the following motif in each individual spatio-temporal history:

$$
\text { organize }=\{\ldots, p A, \underline{p A}, \ldots\}
$$

It is to be noted that a search involving several parameters can be summarized by searching for a quite simple motif of two spatio-temporal states.

- Do some individuals register when they arrive at the conference? The individuals who have not registered for the conference are unknown to the system, they do not possess an identity with regard to the workspace. These individuals obtain an identity as soon as they are registered as participants. The individuals who registered beforehand do not yet possess a spatiality but already have an identity. Based on this observation, it is possible to search for the following motif:

$$
\text { registered on the spot }=\{\underline{p A}, \ldots\}
$$

In this way, all the individuals arriving at the conference and registering directly at the conference are identified. Their spatio-temporal story only begins when they have registered on the spot.

- Who are the individuals who participate in the entire conference? An individual who follows the conference in its entirety will have a continued presence that is entirely located in the portion of space-time defined by the conference workspace. The search for individuals attending the entire conference means searching for the individuals for whom all the possible active states of presence follow one another.

$$
\text { participation }_{\text {complete }}=\forall \text { Hold }- \text { at }(\underline{p W}, x) \rightarrow\{\ldots, \text { hold }- \text { at }(\underline{p A}, x), \ldots\}
$$

This situation corresponds, for an individual, participating in the entire conference, to having a spatio-temporal state of presence located in the space attributed to the conference for each instant that the conference is active. 
The study of the movements of each individual with regard to the conference can be conducted by searching for a particular motif in the succession of spatio-temporal states. As can be seen from Equations (27)-(29), the successions sometimes representing complex semantics are limited to the search for particular spatio-temporal states. We also think that a final user could easily describe his own requests for semantics that are sometimes complex in the motifs of spatio-temporal states.

\subsection{Requests Relative to Two Individuals with Regard to A Workspace}

The study of the dynamics of movement of individuals in relation to the same workspace also takes place by means of the descriptions of the relations these individuals have with regard to the same workspace. The interrogations in terms of spatio-temporal relations mainly concern the fact of knowing if individuals have met each other. This analysis of the spatio-temporal connection constitutes the basis for a lot of areas, such as, for example, epidemiology or criminology. For the two examples cited above, the location of the contact between the individuals is not always the most important information. The interest of this sort of analysis concerns more the type of contact (knowing whether the contact led to a change of direction or behavior) and the duration of the contact (sufficient or not for transmitting information or disease). As previously stated, we do not present an exhaustive list of possible requests through the use of life and motion configurations or the succession of spatio-temporal states but rather an idea of their interest for the search for the semantics in a dataset.

\section{- Who are the individuals who have been in contact with individual A during the conference?}

This request consists of searching for all the individuals who shared the spatial position of individual A during a given moment. Here we consider a distance buffer below for which two individuals are considered as being in an equal position.

$$
\operatorname{contact}_{A-x}=\forall x \rightarrow\{\ldots, \underline{p A p x}, \ldots\}
$$

The search for contacts is limited to finding the spatio-temporal state relations of co-presence between individual A and any other individual $x$. The contact duration can be studied taking account of the duration of the spatio-temporal state or the number of states of the same type that can be found in the life and motion configurations of the movements of the two individuals.

\section{- Who are the individuals that left the conference before the arrival of individual A?}

This request implies a temporal precedence between the action of participating in the conference and the arrival of individual A. This type of request is typically managed by using a temporal antecedence reasoning operator. The motif to be sought in the set of life and motion configurations proposed between individual A and all the other individuals is limited to the search for two types of relation between successive spatio-temporal states.

$$
\text { departure } A<\text { arrival } x=\forall x \rightarrow\{\ldots, \underline{n p A n p x}, \underline{n p A p x}, \underline{n p A} \text { ? } x\}
$$

All the life and motion configurations which end as presented in Equation (34) concern individuals who arrived at the conference after the departure of individual A. In a situation where the individuals who arrived after the departure of A are carriers of information, this can only be transmitted to individual $\mathrm{A}$ at the place where the conference occurs.

These few examples take account of the interest of using life and motion configurations in the search for semantics in a spatio-temporal dataset restricted to a workplace. As previously explained, a workspace can be defined in relation to a zone of visibility or spatiality of an event. This can evolve over time, the search for motifs in a set of life and motion configurations helps to take account of all the variability of the workspace. 


\section{Application to the Data of a Location-Based Social Network}

The technology of geolocalized social networks has evolved considerably over the last few years. Thanks to the willingness of users to share their position in exchange for dedicated services, we currently have large spatially and temporally localized datasets. Although many questions relative to the right to privacy are raised by the use of this data, we can nonetheless use datasets that respect anonymity and are free to use. This often involves old set or information on networks that no longer exist. Nevertheless, it is possible to develop analysis on this type of spatio-temporal data in order to conceptualize future analyses relative to the data collected from active geolocalized networks. The extraction of information can also be seen as a stage guaranteeing the anonymizing of data. Indeed, the description of a behavior or journey purpose of one individual is synthetized, or added to other individuals during analysis, in order to define a general movement tendency. In this way, more relevant information is represented for the analysis required but also more generalized with regard to individual behavior which could lead to a person being identified. In the remainder of this section, we will use a random numerical identifier to describe each user. The data used are from the social network Gowalla (now finished). We will work with a dataset covering a 12-month period between 2009 and 2010. This contains more than 6 million check-ins (that is to say, a geolocalized point temporally known and representing the entry of an individual into a given place). Further information relative to this dataset is available in Cho [43]. The dataset has been loaded in a PostGis database and graphically exploited with QuantumGis. The construction of results sets is performed through SQL/PLPGSQL queries.

The application of life and motion configurations to the data of a social network makes it possible to carry out a rapid extraction of information relative to the connections between individuals without having to graphically represent all their trajectories. When the quantity of data collected becomes important, a temporally simultaneous representation of all the positions of the individuals becomes inefficient in terms of the identification of connections, interactions or the identification of successive visits (see Figure 6). This is often the case for data extracted from social networks. In fact, several characteristics that particularly apply to geolocalized social network data are to be taken into account:

- The data are rarely continuous. In accordance with the profile of each user, the frequency of use of the network can vary strongly (from a person discovering the network and only effecting some check-ins in order to test the system to a person engaging in frequent or even addictive use of the network sharing his positions at each movement). By way of comparison, following a mobile phone by means of a GPS application makes it possible to identify positions at time intervals that are often fixed with great frequency;

- The data are partial. The information about the presence of a person in a place cannot be completely defined by the sole use of a check-in system. When it is effected, the check-in only provides information about the entry of a person into a place. The duration, instant and position of exit from the place remain unknown. According to the type of place, we can suppose that there is a strong possibility of presence during a certain time period (in a cinema, for example) while a check-in into certain places will not supply a lot of location information (in public transport). The position supplied is assimilated to a point while the location to which the check-in refers is a network or element in movement.

Based on these two assessments, we notice that an individual who does not check-in very frequently remains in existence for the system despite the lack of knowledge about his location. It remains necessary, however, to establish the links between users (as previously explained) during these phases of non-presence in the network. The objective of using the spatio-temporal states and life and motion configuration model is to be able to describe the continuous spatial and temporal evolution of these individuals based on data that are conceptualized in a discreet way and with variable frequency. 
In order to visually determine, for example, whether two individuals could interconnect, a representation of all the trajectories of the individuals can be carried out. This is generated either in 2D by projecting temporal information into the 2D space (see Figure 7), or it is represented in a temporal space as defined by [8], in the two cases the quantity of information rapidly leads to an impossibility to visually extract the information requested. Changing to a connection model such as the life and motion configurations then becomes mandatory.

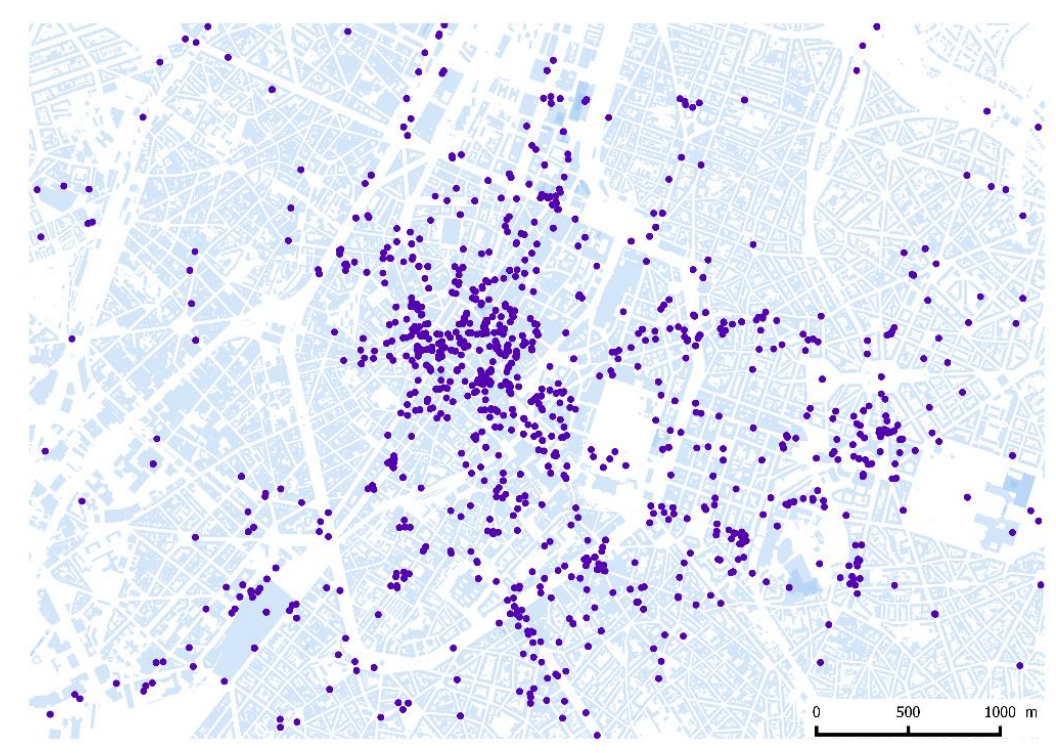

Figure 6. A representation of all the check-ins effected in the center of the city of Brussels, Belgium, during the period of September 2009 to October 2010. The figure shows around 4700 check-ins effected by 742 different users.

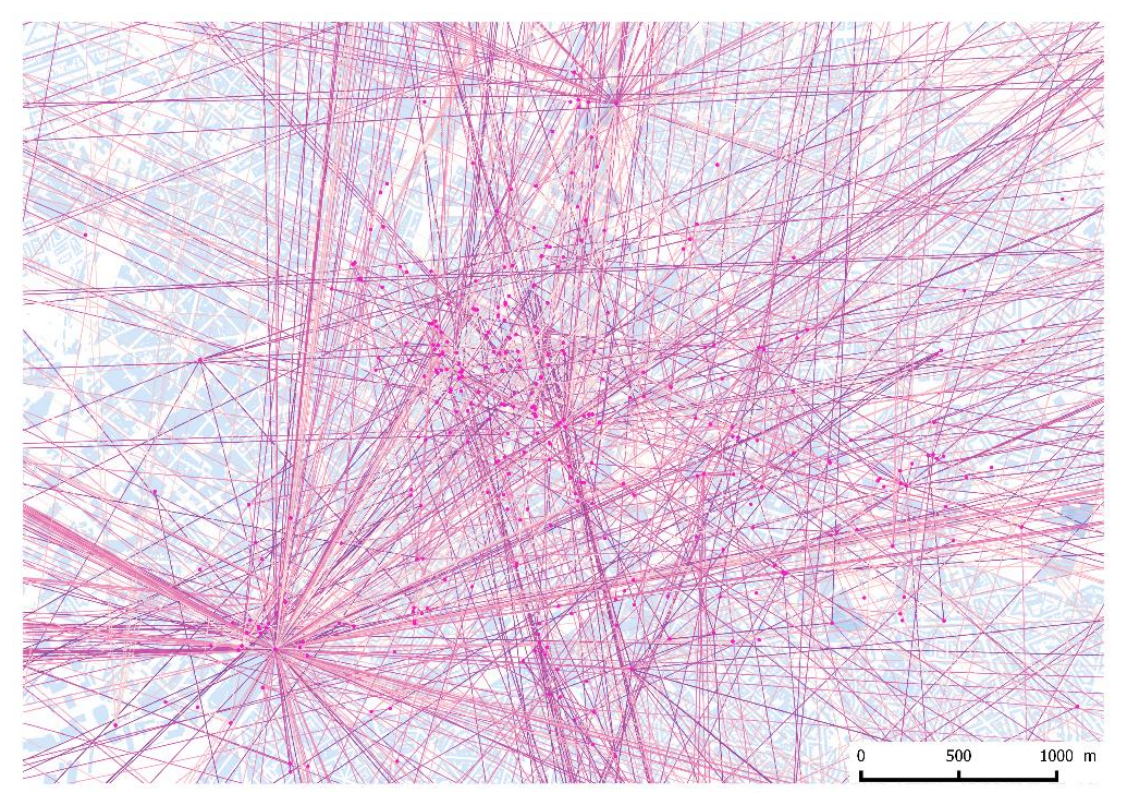

Figure 7. A representation of the trajectories effected by all the individuals who made at least one check-in at the "Midi Station" in Brussels in Belgium. A different color is associated with each username. It is impossible to retrieve information based on such kind of representation. This shows the importance of using a spatio-temporal model as developed here to retrieve information from LBSN datasets. 
In the same way as already established in Section 5, hereafter we detail the way a spatio-temporal state is associated with each situation of existence or presence on the network in order to be able to model this type of dataset.

- $\quad p A$ : Individual $\mathrm{A}$ is spatially located in the space of a place whose activity defining the place is not currently in progress at this given moment. That means that the person has effected a check-in into a place where the activity is past or will occur in the future. This type of situation will only be modeled if the semantic information relating to the places such as defined in the social media is sufficiently detailed to allow this distinction. It involves, for example, a check-in in an external festival location. Except for the duration of the festival (where temporary installations show the existence of a festival), the place where this takes place has no particular characteristic making it possible to define it as a festival location (the temporary installations are dismantled after the festival).

- $\quad n p A$ : Individual $\mathrm{A}$ is temporally existent during the activity of the workspace but not spatially located in the space defined by it. With regard to all the possible workspaces, an individual will always be absent for these once he has been located in another workspace. The relations between workspaces and the consequence of the state of presence or non-presence of an individual can be studied but are outside the scope of this study.

- $\quad p A$ : Individual A is temporally and spatially present during the activity of the workspace. It is this situation that we will encounter most in the analysis we will carry out thereafter.

- $\quad n p A$ : Individual A is not spatially located in the workspace but is temporally present during the activity of the workspace. This situation is that of a person who is active on the network, located in other workspaces than that for which we are calculating his presence.

Based on the definition of this work context, it is then possible to carry out a set of analyses aiming to determine and characterize the movements of an individual in space and time. The calculation of the relations between spatio-temporal states is not possible for such a quantity of data. The comparison is effected based on an individual against all the others. A prior temporal selection makes it possible to identify the relations for which the individuals compared are non-existent, i.e., situations for which their identity is not yet known to the system. In this way, the relation between spatio-temporal states is calculated only for the period temporally covering the existence of their identity. We then arrive at the same possibilities for requests as expressed in Chapter 5.

In the following part, we will detail some possible requests related to the presence of one or several individuals in the workspaces linked to the social network.

\section{- Which individuals have visited the Brussels Midi Station at the same time as individual X?}

This search for persons who share a common portion of space-time within a place is interesting in the context of connections between individuals. The result of this request will provide a set of groups of persons who are situated in the space defined by the station and also at the same moment as the person being compared. The result will not only be a set of persons but a succession of temporal periods during which it is possible to search for common presences with regard to individual X. As soon as individual $X$ does not possess any continued presence within the station, the result will be fragmented equally taking equal account of the period of presence and individual $X$. Note that it is possible for the individual to be alone during a present period in the workspace, consequently this time interval will not show connections.

$$
\underline{p X p ?}=\{\underline{n p X *}, \underline{p X p ?}, \underline{n p X *\}}
$$

In Equation (35), the symbol "** signifies that all the possibilities of states of existence are taken into account. The character "?" represents all the individuals searched for. Figure 8 proposes a spatial representation of the identified trajectories of individuals who shared a presence with individual $X$ in the space dedicated to the station. 
As well as the visual representation, we can collect more detailed information on the connections between individuals in the temporal windows of presence of individual $X$ in the station (see Table 1). In the following example, individual $X$ possesses username 57390. It is on the basis of his presence that the request is applied and that the connected persons are searched for. As we can see, for a window of presence, there has been no correspondence. That signifies that at that time, no other user of the network effected a check-in in the same workspace.

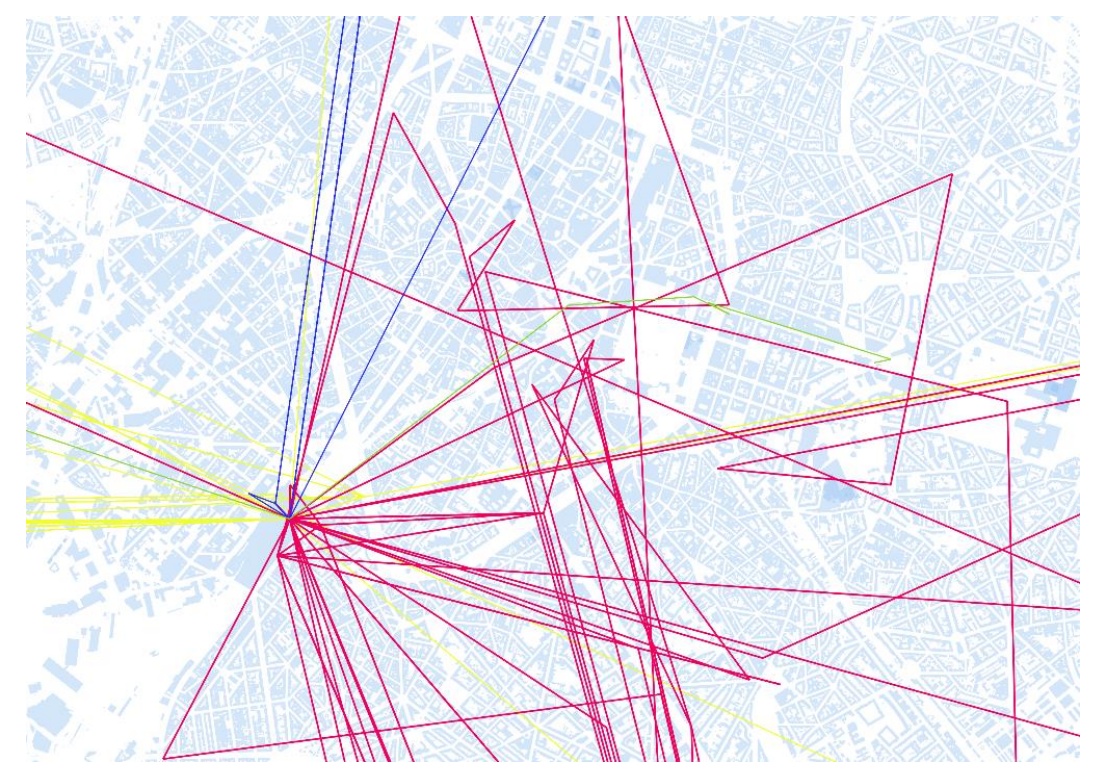

Figure 8. Visualisation of the trajectories of individuals who have been simultaneously spatially and temporally present in the "Midi Station" with the compared individual. The trajectory of the compared individual is represented in red, the three other colors represent the three persons connected to the first individual.

Table 1. Usernames, and temporalities of individuals connected to the comparison individual with the username 57390 during his phases of presence in the "Midi Station". In the example, a temporal tolerance of $15 \mathrm{~min}$ is applied to the check-in.

\begin{tabular}{ccc}
\hline uID & Date-Time & Spatio-Temporal State Relation \\
\hline 57390 & 20 October 2010 6.52 AM & $\underline{\text { p57390p54021 }}$ \\
54021 & 20 October 2010 6.45 AM & \\
- & - & $\underline{\text { p57390np* }}$ \\
57390 & 7 October 2010 6.30 AM & $\underline{\text { p57390p109759 }}$ \\
- & - & $\underline{\text { p57390p119817 }}$ \\
57390 & 22 September 20106.50 AM & \\
109759 & 22 September 2010 6.37 AM & \\
119817 & 22 September 2010 6.25 AM & \\
\hline
\end{tabular}

As previously explained, use of check-in data only provides information about a workspace, consequently, in order to be able to calculate temporal periods covered, we consider a period of presence of $15 \mathrm{~min}$ from the time an individual checks in.

\section{- What arethe most visited places by individuals entering into the town by means of the station?}

Considering the station as an entry point into the town can be understood by the fact that it serves as a main connection point with Brussels airport, which serves the town. Following this, we 
will suppose that the persons who effect the first check-in of their succession in the "Midi Station" enter the town in this way. In addition, the station does not constitute an attractive place to visit in itself. Based on this theory, it is then possible to look at all the workspaces visited by all the individuals. This first classification already supplies information relative the places visited and an idea about their frequentations. It is then possible to see if an order emerges with regard to the succession of each visit. In this way, it will be possible by means of the check-ins by a large number of individuals to see whether a "guided visit" emerges for the town. This would be of great importance to tourist companies in order to adapt their guided tours or inform visitors about appropriate guided tours that could satisfy their preferences. A way of discriminating.

Figure 9 shows the count of check-ins effected in each location. From this classification, we can observe that the communication locations are the most represented. The three main stations of the town are the most frequented (in terms of visibility on the social network). After this come the hotels or remarkable buildings of the town (town hall, cult places, etc.). Finally, a high total number of took place in places of activity such as restaurants, clubs or bars. However, they are only slightly represented in Figure 9, because considered individually, they do not represent a high number of check-ins.

$$
\text { visite_type_uid_X }=\left\{\underline{p X}_{\text {Wstation' }} \underline{p X}_{W 2^{\prime}} \underline{p X}_{W 3^{\prime}} \underline{p X}_{W 4}\right\}
$$

Analysis of these successions of visits to places can lead to the definition of a chain of presence in places such as those proposed in Equation (36). These show the successive presences of individual $X$ in different workspaces (Wstation, W2, W3, and W4). The realization of this type of connection chain for each individual whose chain commences by a presence in the workspace of the station makes it possible to carry out possible successions in several places.

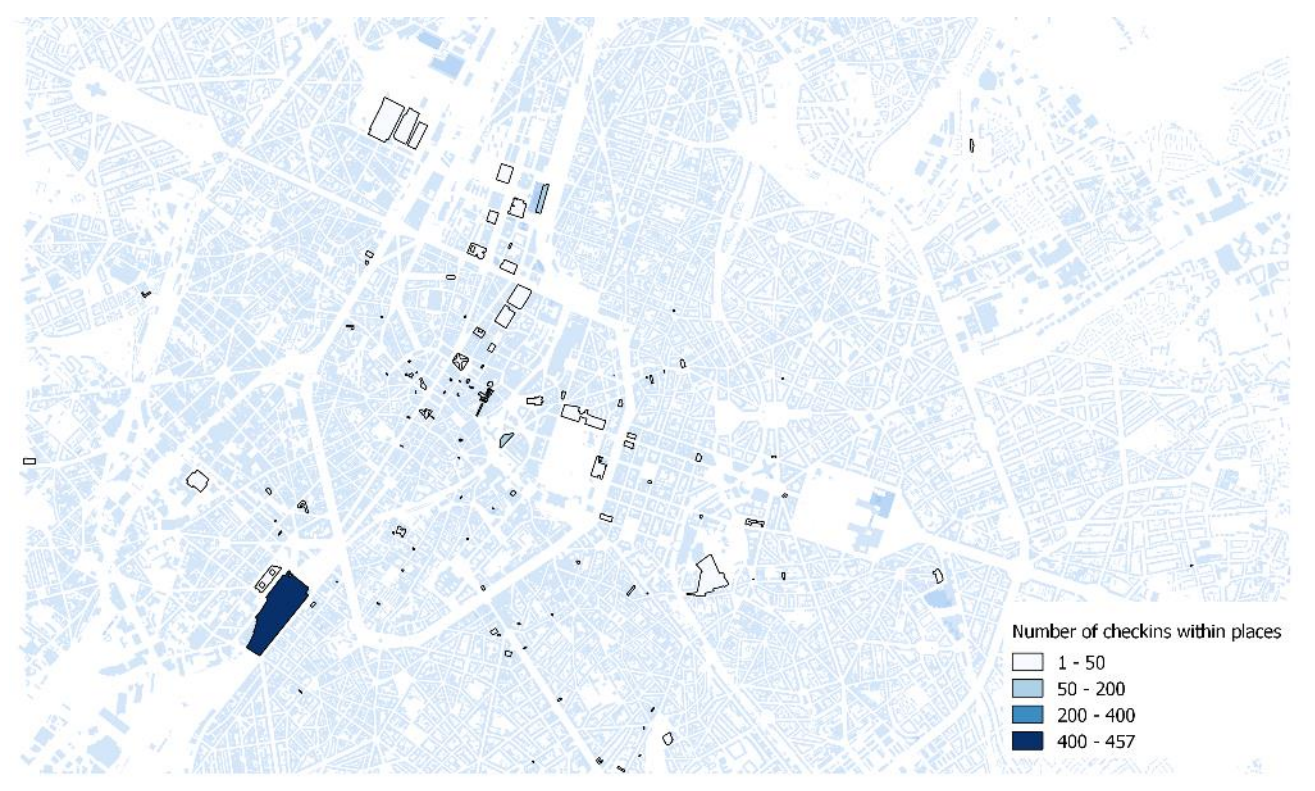

Figure 9. The number of check-ins effected in each location. The locations in which there are more check-ins include stations, hotels, tourist haunts and famous buildings.

\section{Discussion}

Recognizing that current models of spatio-temporal lines of reasoning do not take account of the management of relations to absent or non-existent objects, we propose, in this work, a model of spatio-temporal reasoning constructed on the evolution of the identity of a geographical object. By proposing a complete analysis of all the stages of conceptualization and creation of a geographical object, it is possible for us to formally define a set of spatio-temporal states representing all the 
possibilities of existence of an object. By analyzing these spatio-temporal states with regard to the workspace, it is possible for us to include all the variability of existence and presence of geographical objects. In order to follow the evolution of spatio-temporal relations between geographical objects in time, we also propose a framework of representation by means of life and motion configurations, which open the door to a whole series of analysis possibilities. Through this work, we have shown that it is possible to easily perform searches in a spatio-temporal dataset based on a semantically rich method. More than a search method, we propose a work context that opens the door to a series of supplementary analyses on a dataset restricted to a workplace.

As explained previously, the level of adaptability of the method to various situations in terms of types of data or environmental complexity in which there data evolve is very high. Such as, for example, specific requests for the evolution of persons in a geolocalized social network based on check-ins, we think that to reflect on the spatio-temporal information by means of the use of spatio-temporal states and life and motion configurations can provide a more semantically complete result in terms of the request for this type of information.

The follow-up to this search is devoted to the implementation of a system that automatically calculates the life and motion configurations for a set of information. For now, we are able to calculate the spatio-temporal states from datasets obtained of location-based social networks. In the near future, we will work on a spatio-temporal data structure that fully encompass all the diversity of the life and motion configurations. A particular attention will be dedicated to the performance of queries based on life and motion configuration pattern recognition. Since location based social networks provide big datasets with billions of check-ins, it is necessary to propose a fully optimized system. In addition, we propose to study the possibility of automatically linking semantics such as meeting and crossing to requests made by a final user. The study of the combination of several combined workspaces is also part of our future search objectives. In this way, we hope to define the spatio-temporal relations between several datasets more easily and between datasets with different spatio-temporal granularity. Finally, the combination of life and motion configurations calculated for several individuals evolving in a workspace can lead to the calculation of a chain of connections in order to identify whether an individual A can enter into contact with an individual X, and, if so, by means of what intermediaries?

Author Contributions: Pierre Hallot designed the method, performed analysis, interpreted data, wrote manuscript and acted as corresponding author. Roland Billen supervised development of work, reviewed and edited the manuscript and helped in data interpretation and method evaluation.

Conflicts of Interest: The authors declare no conflict of interest.

\section{References}

1. Khoshafian, S.N.; Copeland, G.P. Object identity. SIGPLAN Not. 1986, 21, 406-416. [CrossRef]

2. Zalta, E. The stanford encyclopedia of philosophy. Coll. Res. Libr. News 2006, 67, 502-520.

3. Hornsby, K.; Egenhofer, M.J. Identity-based change: A foundation for spatio-temporal knowledge representation. Int. J. Geogr. Inf. Sci. 2000, 14, 207-224. [CrossRef]

4. Worboys, M.; Hornsby, K. From objects to events: GEM, the geospatial event model. In Geographic Information Science; Egenhofer, M.J., Freksa, C., Miller, H.J., Eds.; Springer: Berlin, Germany, 2004; Volume 3234, pp. 327-343.

5. Hornsby, K.; Egenhofer, M. Identity-based change operations for composite objects. In Proceedings of the 8th International Symposium on Spatial Data Handling, Vancouver, BC, Canada, 12-15 July 1998; pp. 202-213.

6. Campos, J.; Hornsby, K.; Egenhofer, M. A temporal model of virtual reality objects and their semantics. J. Vis. Lang. Comput. 2003, 14, 469-492.

7. Claramunt, C.; Jiang, B. A representation of relationships in temporal spaces. In Innovations in GIS VII: GeoComputation; Atkinson, P., Martin, D., Eds.; Taylor \& Francis: London, UK, 2000; pp. 41-53.

8. Claramunt, C.; Thériault, M.; Parent, C. A qualitative representation of evolving spatial entities in two-dimensional topological spaces. In Innovations in GIS V; Carver, S., Ed.; Taylor \& Francis: London, UK, 1997; pp. 119-129. 
9. Claramunt, C.; Jiang, B. An integrated representation of spatial and temporal relationships between evolving regions. J. Geogr. Syst. 2001, 3, 411-428. [CrossRef]

10. Egenhofer, M.; Herring, J. Categorizing Binary Topological Relations between Regions, Lines and Points in Geographic Databases; Technical Report 01/1990; University of Maine: Orono, ME, USA, 1990; p. 28.

11. Egenhofer, M.; Franzosa, R.D. Point-set topological spatial relations. Int. J. Geogr. Inf. Syst. 1991, 5, 161-174. [CrossRef]

12. Allen, J.F. Maintaining knowledge about temporal intervals. Commun. ACM Univ. Rochester 1983, 26, 832-843. [CrossRef]

13. Allen, J.F. Towards a general theory of action and time. Artif. Intell. 1984, 23, 123-154. [CrossRef]

14. Fan, Y.T.; Yang, J.Y.; Zhang, C.; Zhu, D.H. A event-based change detection method of cadastral database incremental updating. Math. Comput. Model. 2010, 51, 1343-1350. [CrossRef]

15. Van de Weghe, N.; Cohn, A.G.; De Tre, G.; De Maeyer, P. A qualitative trajectory calculus as a basis for representing moving objects in geographical information systems. Control Cybern 2006, 35, 97-119.

16. Bogaert, P.; van de Weghe, N.; Cohn, A.G.; Wiltox, F.; de Maeyer, P. The qualitative trajectory calculus on networks. In Spatial Cognition V Reasoning, Action, Interaction; Barkowsky, T., Freksa, C., Knauff, M., Krieg-Brückner, B., Nebel, B., Eds.; Springer: Bremen, Germany, 2006; pp. 20-38.

17. Van de Weghe, N.; Kuijpers, B.; Bogaert, P.; de Maeyer, P. A qualitative trajectory calculus and the composition of its relations. Lect. Notes Comput. Sci. 2005, 3799, 60-76.

18. Noyon, V.; Claramunt, C.; Devogele, T. A relative representation of trajectories in geogaphical spaces. Geoinformatica 2007, 11, 479-496. [CrossRef]

19. Noyon, V.; Devogele, T.; Claramunt, C. A formal model for representing point trajectories in two-dimensional spaces. In Proceedings of the 2005 International Conference on Conceptual Modelling ER'05, Klagenfurt, Austria, 24-28 October 2005; pp. 208-217.

20. Vila, L. A survey on temporal reasoning in artificial intelligence. AI Commun. 1994, 7, 4-28.

21. Abraham, T.; Roddick, J. Survey of spatio-temporal databases. Geoinformatica 1999, 3, 61-69. [CrossRef]

22. Galton, A.A. Spatial and temporal knowledge representation. Earth Sci. Inf. 2009, 2, 169-187. [CrossRef]

23. Stock, O. Spatial and Temporal Reasoning; Kluwer Academic Publishers: Dordrecht, The Netherlands, 1997.

24. Pelekis, N.; Theodoulidis, B.; Kopanakis, I.; Theodoridis, Y. Literature review of spatio-temporal database models. Knowl. Eng. Rev. 2004, 19, 235-274. [CrossRef]

25. Keßler, C.; Raubal, M.; Wosniok, C. Semantic rules for context-aware geographical information retrieval. In Smart Sensing and Context; Barnaghi, P., Moessner, K., Presser, M., Meissner, S., Eds.; Springer: Berlin, Germany, 2009; Volume 5741, pp. 77-92.

26. Cai, G. Contextualization of geospatial database semantics for human-GIS Interaction. Geoinformatica 2007, 11, 217-237. [CrossRef]

27. Claramunt, C.; Theriault, M. Toward semantics for modelling spatio-temporal processes within GIS. In Advances in GIS II; Kraak, M.J., Molenaar, M., Eds.; Taylor and Francis: Delft, the Netherlands, 1996; pp. 47-64.

28. Lawson, S.; van der Zee, E.; Daley, L. Spatial language in computer mediated communication. In Exploration of Space, Technology, and Spatiality: Interdisciplinary Perspectives; IGI Global: Hershey, PA, USA; pp. 168-182.

29. Traugott, E. On the expression of spatio-temporal relations in language. In Universals of Human Language: Word structure; Stanford University Press: Palo Alto, CA, USA, 1978; pp. 369-400.

30. Cova, T.; Church, R. Modelling community evacuation vulnerability using GIS. Int. J. Geogr. Inf. Sci. 1997, 11, 763-784. [CrossRef]

31. Hallot, P.; Stewart, K.; Billen, R. Les états spatiotemporels d'existence et de présence. Int. J. Geomat. Spat. Anal. 2015, 25, 173-196. [CrossRef]

32. Galton, A. Towards a qualitative theory of movement. In Spatial Information Theory: A Theorical Basis for GIS; Kuhn, W., Frank, A., Eds.; Springer: Berlin, Germany, 1995; Volume 988, pp. 377-396.

33. Galton, A. A qualitative approach to continuity. In Proceedings of the 5th International Workshop on Time, Space and Movement: Meaning and Knowledge in the Sensible World (TSM'95), Château de Bonas, France, 23-27 June 1995; pp. 17-30.

34. Galton, A. Qualitative Spatial Change; Oxford University Press: Oxford, UK, 2000.

35. Hägerstand, T. Innovation Diffusion as Spatial Process; University of Chicago Press: Chicago, IL, USA, 1968.

36. Hallot, P. L'identité à Travers L'espace et le Temps: Vers une DÉFINITION de L'identité et des Relations Spatio-Temporelles Entre Objets Géographiques. Ph.D. Thesis, University of Liège, Liège, Belgium, 2012. 
37. Freksa, C. Qualitative spatial reasoning. In Cognitive and Linguistic Aspects of Geographic Space; Mark, A.U., Frank, D.M., Eds.; Kluwer Academic Publishers: Dordrecht, The Netherlands, 1991; pp. 361-372.

38. Galton, A. Dominance diagrams: A tool for qualitative reasoning about continuous systems. Fundam. Inf. 2001, 46, 55-70.

39. Billen, R.; Clementini, E. A model for ternary projective relations between regions. In Proceedings of 9th International Conference on Extending Database Technology, Heraklion, Greece, 14-18 March 2004; pp. 310-328.

40. Hallot, P.; Billen, R. Generalized life and motion configuration reasoning model. In Proceedings of the International Workshop on Moving Objects: From Natural to Formal Language, Collocated with GIScience 08, Park City, UT, USA, 23 September 2008.

41. Keet, C.M.; Fernández-Reyes, F.; Morales-González, A. Representing mereotopological relations in OWL ontologies with OntoPartS. In The Semantic Web: Research and Applications; Simperl, E., Cimiano, P., Polleres, A., Corcho, O., Presutti, V., Eds.; Springer: Berlin, Germany, 2012; Volume 7295, pp. 240-254.

42. Stewart, K.; Fan, J.; White, E. Thinking about space-time connections: Spatiotemporal scheduling of individual activities. Trans. GIS 2013, 17, 791-807. [CrossRef]

43. Cho, E.; Myers, S.; Leskovec, J. Friendship and mobility: User movement in location-based social networks. In Proceedings of the SIGKDD Conference on Knowledge Discovery and Data Mining, San Diego, CA, USA, 21-24 August 2011.

(C) 2016 by the authors; licensee MDPI, Basel, Switzerland. This article is an open access article distributed under the terms and conditions of the Creative Commons Attribution (CC-BY) license (http:/ / creativecommons.org/licenses/by/4.0/). 